\title{
Evolución del marketing 1.0 al 4.0
}

\author{
Suárez-Cousillas, Tara \\ Universidade da Coruña \\ tara.suarez@udc.es
}

Forma de citar esta reseña: Suárez, T. (2018). Evolución del marketing 1.0 al 4.0, Redmarka. Revista de Marketing Aplicado, vol 01, núm. 022, 209-227. https://doi.org/10.17979/redma.2018.01.022.4943

\section{Resumen}

El nacimiento de Internet, el uso cotidiano de smartphones, la geolocalización y demás avances tecnológicos, ofrecen la posibilidad de que las empresas contacten y se comuniquen con un mayor número de personas, lo cual puede incidir en su posicionamiento en el mercado.

Entre otros factores, el uso de las nuevas tecnologías de la información y comunicación ha cambiado el funcionamiento de las empresas, el comportamiento del consumidor y también el concepto de marketing con relación a cómo eran entendidos a principios del siglo pasado. En una época donde las empresas sólo se esforzaban económicamente en el desarrollo del producto, se llega a un momento focalizado en el consumidor donde todos los esfuerzos de las empresas están dirigidos a detectar las necesidades de los clientes, incluso antes de que ellos las conozcan. Se pasa de un enfoque de producción a un enfoque de marketing.

El objeto de la presente investigación, de carácter aproximativo, consiste en efectuar un análisis de la evolución del marketing, desde el 1.0 al 4.0. Los objetivos planteados son: analizar, de forma cuantitativa, la producción científica sobre Marketing, en sus diferentes fases, y conocer cuáles son las 
áreas de investigación con mayor producción científica en referencia al punto anterior en Scopus. La metodología aplicada se centra en la búsqueda de producción científica en la base de datos Scopus para el período comprendido entre el 2014 y 2018.

Palabras clave: Marketing, evolución, marketing digital, marketing 4.0, big data, predicción.

\section{Abstract}

The birth of the Internet, the daily use of smartphones, geolocation and other technological advances, offer the possibility for companies to contact and communicate with a greater number of people, which can affect their positioning in the market.

Among other factors, the use of new information and communication technologies has changed the functioning of companies, consumer behavior and the concept of marketing in relation to how they were understood at the beginning of the last century. In a time when companies only made economic efforts in the development of the product, it reaches a time focused on the consumer where all the efforts of the companies are aimed at detecting the needs of customers, even before they know them. It goes from a production approach to a marketing approach.

The object of the present investigation, of approximate character, consists in carrying out an analysis of the evolution of marketing, from 1.0 to 4.0. The proposed objectives are: to analyze, quantitatively, the scientific production on Marketing, in its different phases, and to know which the research areas with the highest scientific production about the previous point in Scopus are. The methodology applied focuses on the search for scientific production in the Scopus database for the period between 2014 and 2018.

Keywords: marketing, evolution, digital marketing, marketing 4.0, big data, prediction. 


\section{MARCO TEÓRICO}

El presente estudio se dividirá en 4 partes. El punto 1 es el marco teórico donde, a través de una breve introducción, se enmarcará la evolución del marketing. Dentro de esta introducción, delimitados en subapartados, se definirá el concepto de marketing según diversos autores $y$, en el segundo subapartado, se analiza la evolución del marketing desde el 1.0 hasta el 4.0, introduciendo el Big Data en la última fase de la evolución.

El punto 2 de Metodología, se hace una búsqueda con relación a los parámetros, Marketing, Marketing 1.0, Marketing 2.0, Marketing 3.0, Marketing 4.0, Marketing 3.0 y Big Data, Marketing 4.0 y Big Data, Marketing y Big Data, en la base de datos Scopus. El apartado de Metodología expone el alcance de este estudio. El apartado de resultados, el punto número 3 , recoge el análisis expuesto en la metodología.

Por último, el punto número 4, engloba las discusiones y conclusiones obtenidas.

\subsection{INTRODUCCIÓN}

El marketing, al igual que otras disciplinas, ha ido adaptándose a los diferentes avances de la humanidad, tecnologías, conocimientos y necesidades.

En la España de los años sesenta y cincuenta, el foco de atención para una empresa se centraba en la producción, "era una época en la que todo lo que se producía se vendía" (De Vicuña Ancí, 2018). Pese a que también había actividad comercial y publicidad, no tenía nada que ver con el marketing. Con una mentalidad centrada en la producción, las empresas no se preocupaban de los clientes, confiaban en que con la calidad de los productos estos se vendieran solos, a lo sumo, se le daba importancia al envase, pues era la primera imagen que se veía del producto. Esto ocasionó que la innovación se centrase en mejorar el producto y no las ventas.

Con el paso del tiempo, el marketing ha evolucionado a un marketing estratégico, las empresas empiezan a prestar atención a las necesidades de 
sus clientes, usa técnicas de segmentación, estrategias para abordarlos.... Y obtener así un mayor impacto en ellos. Se partía de un enfoque de producción para llegar a uno de marketing tal y como se conoce hoy en día, donde el cliente es lo primero, se le consulta sobre sus necesidades o deseos, y luego se produce o comercializa el producto. Gracias a Internet, al comercio electrónico y las nuevas tecnologías se puede hablar de marketing digital. El cliente es la razón de ser de la organización.

Hay diferentes definiciones para el término marketing dadas por los distintos expertos y profesionales, pero, al igual que lo hace la tecnología, el concepto ha evolucionado. Mediante una revisión bibliográfica, se trata de proporcionar a través de este trabajo una visión de esta evolución partiendo de la definición de Marketing.

\subsection{EL CONCEPTO}

La que se entiende hoy en día por Marketing es el resultado de una evolución del término acompañada del progreso de la humanidad.

Diferentes autores han tratado de definir el Marketing. Uno de ellos ha sido, Santesmases, M. (2012) señala que "el marketing se concibe actualmente como medio para establecer relaciones de intercambio estables y duraderas con los clientes, que sean mutuamente satisfactorias".

Según Martin, J., y Ocampo, J.A. (2003), con la aparición del capitalismo a finales de la Edad Media en Europa surge el proceso contemporáneo de internacionalización, con una nueva actitud científica y cultural que reencarnó el Renacimiento y la formación de grandes naciones europeas y sus imperios. Pese a no estar completo, el único fenómeno histórico con alcance realmente global ha sido la expansión del capitalismo.

La American Marketing Association (AMA) define el Marketing como: "Marketing is the activity, set of institutions, and processes for creating, communicating, delivering, and exchanging offerings that have value for customers, clients, partners, and society at large" (AMA, 2018). (El marketing 
es la actividad, el conjunto de instituciones y los procesos para crear, comunicar, entregar e intercambiar ofertas que tienen valor para los clientes, clientes, socios y la sociedad en general).

El padre del marketing, Philip Kotler, define el Marketing como la "filosofía según la cual el logro de las metas de la organización depende de conocer las necesidades y los deseos de los mercados meta, así como de ofrecer la satisfacción deseada de mejor manera que los competidores." (Kotler, P. y Armstrong, G., 2017, p. 10). "El concepto de marketing adopta una perspectiva de afuera hacia dentro, la cual se enfoca en satisfacer las necesidades del cliente para obtener utilidades." (Kotler, P. y Armstrong, G., 2017, p. 11). Se podría decir que "en el ámbito de la empresa el marketing se basa en construir las técnicas necesarias para colocar y vender los productos en el mercado." (Sixto, J., 2016).

Por otra parte, para Morales, Aguilar y Marín (2016), el año 1989 fue el punto de inflexión de la globalización y el marketing. Los ordenadores personales habían ingresado en el mercado de masas, y a principios de la década de los noventa, nacía Internet como complemento de gran potencial. Así, a esta red de ordenadores interrelacionados, se unía una red humana de personas interconectadas. La información en red permitía una mayor interacción entre personas y facilitaba la divulgación boca a boca de la información compartida. La información se convirtió en algo omnipresente. (Kotler, Kartajaya y Setianwan, 2013).

\section{3¿CÓMO HA EVOLUCIONADO? DEL MARKETING 1.0 AL 4.0}

El Marketing 1.0 surge en una época donde la producción en masa (tras la Revolución Industrial) estaba en pleno alce y no había criterios para el consumo, simplemente se consumía lo que el mercado ofrecía sin importar las diferentes características de los productos o servicios. Las empresas invertían únicamente en el desarrollo del producto. Una de las características a destacar en esta fase es la comunicación unidireccional, donde no se escuchaba a los clientes y eran sólo las empresas las que aportaban información. 
Con un enfoque más centrado en el cliente, surge el Marketing 2.0, donde el cliente presta atención a las diferentes ofertas y toma decisiones. Las empresas buscan tanto satisfacer al cliente como retenerlo, es decir, la fidelización. La conversación entre marca y consumidor empieza a funcionar gracias a centros de atención al cliente y la web 2.0 donde los medios digitales y sociales permiten la retroalimentación de las experiencias de los usuarios al igual que la creación de contenido.

Las características de esta nueva versión del marketing son el uso de tecnologías de la información (herramientas y métodos tecnológicos de distribución de a información), la búsqueda del posicionamiento corporativo del producto con la diferenciación como concepto clave y todo ello sin perder de vista el objetivo de satisfacer y retener al cliente.

El marketing 2.0 está muy unido al Big Data, y según Kotler, Kartajaya y Setianwan:

El social media marketing (marketing 2.0) surge en la actual era de la información, basada en las tecnologías de la información. La tarea del marketing ya no es tan sencilla. Los compradores de hoy están bien documentados y pueden comprar fácilmente diversas ofertas de productos similares. Es el consumidor quien define el valor del producto. Las preferencias de los consumidores difieren mucho de unos a otros. La empresa debe segmentar el mercado y desarrollar un producto superior para un segmento objetivo específico dentro del mercado. La regla de oro de: el cliente manda, funciona bien para casi todas las empresas. (Kotler, Kartajaya y Setianwan, 2013, p. 19-20):

El Marketing 3.0, según Kotler, P., Kartajaya, H., y Setiawan, I. (2012) es:

El marketing 3.0 cree que los consumidores son seres humanos integrales, y que todas sus necesidades y deseos deben ser siempre atendidos. Por lo tanto, el marketing 3.0 complementa el marketing emocional con uno espiritual. En el marketing 3.0 las empresas se 
diferencian entre sí por sus valores. (Kotler, Kartajaya \& Setiawan, 2012, p. 11).

En esta fase es donde el cliente, además del papel adquirido anteriormente, evalúa otros puntos ofertados por las marcas como la responsabilidad social. Los clientes, aparte de satisfacer sus necesidades, buscan la satisfacción de sus sentimientos y valores. Esto lleva a que las empresas vendan valores asociados a sus marcas buscando el impacto en su público objetivo.

En un mundo donde los gustos de los consumidores y sus deseos y necesidades cambian constantemente, siempre existen nuevos retos y desafíos para las empresas y, por ello, para el marketing, el cual se debe renovar cada vez que encuentre un desafío nuevo y así poder ser más eficiente en alcanzar sus objetivos.

A modo resumen, la siguiente tabla muestra una comparación entre el Marketing 1.0, 2.0 y 3.0 mediante diferentes características

Tabla 1: Comparación del Marketing 1.0, 2.0 y 3.0

\begin{tabular}{|l|l|l|l|}
\cline { 2 - 4 } \multicolumn{1}{l|}{} & $\begin{array}{l}\text { MARKETING 1.0 } \\
\text { Marketing } \\
\text { centrado en el } \\
\text { producto }\end{array}$ & $\begin{array}{l}\text { MARKETING 2.0 } \\
\text { Marketing } \\
\text { centrado en el } \\
\text { consumidor }\end{array}$ & $\begin{array}{l}\text { MARKETING 3.0 } \\
\text { Marketing } \\
\text { centrado en los } \\
\text { valores }\end{array}$ \\
\hline Objetivo & $\begin{array}{l}\text { Vendedor } \\
\text { productos }\end{array}$ & $\begin{array}{l}\text { Satisfacer y } \\
\text { retener a los } \\
\text { consumidores }\end{array}$ & $\begin{array}{l}\text { Hacer de este } \\
\text { mundo un } \\
\text { mundo mejor }\end{array}$ \\
\hline $\begin{array}{l}\text { Fuerzas } \\
\text { Propulsoras }\end{array}$ & $\begin{array}{l}\text { Revolución } \\
\text { industrial }\end{array}$ & $\begin{array}{l}\text { Tecnologías de } \\
\text { la información }\end{array}$ & $\begin{array}{l}\text { Nueva ola } \\
\text { tecnológica }\end{array}$ \\
\hline $\begin{array}{l}\text { Percepción } \\
\text { del mercado } \\
\text { por la } \\
\text { empresa }\end{array}$ & $\begin{array}{l}\text { Mercado de } \\
\text { masas. } \\
\text { Consumidores } \\
\text { con necesidades } \\
\text { físicas }\end{array}$ & $\begin{array}{l}\text { Consumidores } \\
\text { más inteligente } \\
\text { con mente y } \\
\text { corazón }\end{array}$ & $\begin{array}{l}\text { Ser humano } \\
\text { integral con } \\
\text { mente, corazón } \\
\text { yespiritu }\end{array}$ \\
\hline $\begin{array}{l}\text { Concepto } \\
\text { fundamental } \\
\text { de marketing }\end{array}$ & $\begin{array}{l}\text { Desarrollo de } \\
\text { producto }\end{array}$ & Diferenciación & Valores \\
\hline $\begin{array}{l}\text { Directrices de } \\
\text { marketing } \\
\text { corporativas }\end{array}$ & $\begin{array}{l}\text { Especificaciones } \\
\text { del producto }\end{array}$ & $\begin{array}{l}\text { Posicionamient } \\
\text { o corporativo y } \\
\text { de producto. } \\
\text { Misión, visión y y } \\
\text { valores }\end{array}$ & $\begin{array}{l}\text { Proposiciones } \\
\text { de valor }\end{array}$ \\
\hline $\begin{array}{l}\text { Propuesta de } \\
\text { valor }\end{array}$ & Funcional & $\begin{array}{l}\text { Funcional y } \\
\text { emocional }\end{array}$ & $\begin{array}{l}\text { Eincional, } \\
\text { emocional y } \\
\text { espiritual }\end{array}$ \\
\hline $\begin{array}{l}\text { Interacción } \\
\text { con los } \\
\text { consumidores }\end{array}$ & $\begin{array}{l}\text { Transacciones } \\
\text { uno a uno }\end{array}$ & $\begin{array}{l}\text { Relaciones uno } \\
\text { a uno }\end{array}$ & $\begin{array}{l}\text { Colaboración } \\
\text { entre muchos }\end{array}$ \\
\hline
\end{tabular}

Fuente: Kotler, Kartajaya y Setianwan, p. 21, 2013

La última versión que tenemos a día de hoy es el Marketing 4.0 donde, según Kotler, P., Kartajaya, H., y Setiawan, I. (2017), el objetivo es la confianza y 
fidelización del cliente, usando del marketing tradicional lo mejor e interactuando con la parte online del marketing digital.

El Marketing 4.0 y el ámbito digital van de la mano, la conexión ininterrumpida a la Red, el aumento de la capacidad de trabajo diario al igual que la comunicación directa marca-consumidor, propician su expansión. En un entorno en el cual los clientes disponen de menos tiempo, donde se quieren las cosas inmediatamente (Martínez-Fernández, Valentín-Alejandro, 2016) y lleno de posibilidades, las empresas deben esforzarse en enviar el mensaje que el cliente realmente quiere oír. Podría ser como una era de investigación de mercados constante donde, gracias a los distintos avances (geolocalización, Big Data,...), es posible una monitorización 24 horas de los clientes actuales y potenciales. Actualmente la competencia es a nivel global por lo que se debe buscar una comunicación $360^{\circ}$ en la cual la conversación es directa, se tienen en cuenta los canales on y off-line y las redes sociales juegan un papel muy importante. Son los clientes los que realmente tienen algo que decir, generan contenido en sus redes sociales y en los perfiles digitales de las empresas. Aquí, las marcas tienen que predecir (apoyarse en el Big Data podría ser una opción) las tendencias, saber qué es lo que los clientes van a consumir, identificar nuevos escenarios posibles.

Marisa Martín (2016) también apuesta por la evolución del Marketing 3.0 al Marketing 4.0, acompañada del avance tecnológico y las redes sociales. Considera que el Marketing 3.0 o mercadotecnia es como "conducir a un individuo a la adquisición o compra de un bien o servicio, que se sienta satisfecho y por ello repita la experiencia y lo recomiende a su círculo social". Es más, considera que el marketing actual está más centrado en "anticipación o predicción de las acciones de un individuo colectivo con el objetivo de que realice o recomiende la adquisición de un bien o servicio, repita la experiencia y comparte leche vivencia en el entorno global". 
Tabla 1: Del Marketing 1.0 al 4.0

\begin{tabular}{|c|c|c|c|c|}
\cline { 2 - 5 } \multicolumn{1}{c|}{} & MARKETING 1.0 & MARKETING 2.0 & MARKETING 3.0 & MARKETING 4.0 \\
\hline FOCO & Producto & Consumidor & Emociones/Valores & Predicción y Anticipación \\
\hline OBJETIVO & Vender & Satisfacer y Retener & Construir un Mundo Mejor & Identificar Tendencias \\
\hline FUERZA & Revolución & Tecnologías de & Nueva Era & Big Data \\
PROPULSORA & Industrial & la Información & Tecnología & \\
\hline CONEXIÓN & Sin conexión & Información y Personas & Conocimiento & Inteligencia \\
\hline
\end{tabular}

Fuente: Marisa Martin Jiménez

En esta nueva etapa de la evolución del marketing, la aparición del Big Data y la analítica de datos aporta una nueva oportunidad para las empresas y en especial para los departamentos de marketing, pues, pueden obtener información realmente valiosa de sus clientes actuales y futuros a gran escala, sus gustos, preferencias, intereses... el marketing en esta era digital se basa en los gustos, deseos y tendencias que recopilan a través de distintos canales (redes sociales, productos wearables, internet de las cosas..) y la oportunidad está en saber interpretar todos estos para llegar al objetivo.

El Big Data, según Mayer y Cukier (2013) es un conjunto de datos masivos, de una estructura grande, más variada y compleja que supera la capacidad del software convencional, como por ejemplo las bases de datos, para ser capturados, administrados y procesados en un tiempo razonable. También se le denomina como macrodatos, datos masivos, inteligencia de datos o datos a gran escala. Big Data se refiere a nuestra nueva habilidad para hacer cálculos respecto a una gran cantidad de información, analizarla al instante, y sacar conclusiones a veces sorprendentes.

Otra definición, según Krishnan (2013) así como Philip Chen y Zhan (2014), del concepto Big Data es, que se caracteriza por tres variables conocidas como las 3 Vs: Volumen, Velocidad y Variedad. Donde el Volumen hace referencia a la cantidad de datos generados continuamente en un espacio de tiempo determinado; Velocidad, a la rapidez de entrada y salida con la cual éstos fluyen a través de distintos canales; y Variedad, a los diferentes formatos y fuentes en que éstos se encuentran. Lo plantean a modo de definición base a la cual se le pueden añadir más variables: Veracidad, Validez y Volatilidad 
(Normandeau, 2013), pero es Valor, la cuarta V, aquella que permite extraer de los datos (Tabares, y Hernández, 2014).

Un hándicap de los datos recogidos es que pueden estar estructurados o no, aunque no influya para el fin de almacenarlos, si lo hace para adoptar decisiones, analizar comportamientos o estrategias de negocios. Se necesitan los datos estructurados pues, de no ser así, la toma de decisiones sería muy pobre.

De hecho, McAfee y Brynjolfsson (2012) concluyen un estudio, efectuado al respecto, con la aseveración de que la explotación de los nuevos y espectaculares flujos de información pueden mejorar realmente el desempeño de la empresa. Sin embargo, será necesario cambiar su cultura de toma de decisiones, por lo cual propone que las decisiones controladas por los datos tienden a ser mejores decisiones.

El objetivo fundamentalmente consiste en conocer el comportamiento y predecirlo; anticiparse a él, lo cual es sumamente importante para todo tipo de empresas. El Big Data proporciona una gran cantidad de respuestas, es como un punto de partida y desde ahí se estructuran y prueban los datos. Primordialmente se realizan estudios de mercado para conseguir resultados a partir de una muestra de la población, pero es ahí donde se observa la gran importancia del Big Data, ya no es necesaria una muestra, hay acceso a todo el mundo a través de la Red. Como consecuencia, abarata costes para las organizaciones, permite diferentes análisis sin verse acotados por el tamaño de la muestra y se pueden crear escenarios, caros y complejos que, en la realidad, serían imposibles de probar (Torres, 2012). También accede a un tratamiento más ágil y más rápido de los datos, con el inconveniente de la vertiginosa modificación de la información al recoger datos de miles de millones de personas con sus propias creencias y opiniones.

La recolección de datos, tal como dice Mayer (2013), comienza a ser tan barata que no se necesitarán las muestras para estudiar un hecho, toda vez que se podrá analizar el conjunto total de datos y serán más precisos, más baratos y 
más inesperados. Por consiguiente, debemos de estar dispuestos a aceptar resultados basados en la correlación sin entender completamente el por qué, la causa. Además, señala la gran importancia del Big Data en el éxito de compañías como Facebook, Gmail, Netflix o Amazon. Con relación a ello, considera que "ni Facebook ni Google existirian sin los datos masivos. Así, Amazon ha obtenido un tercio de sus ganancias de productos que la gente compra por su sistema de recomendaciones, que consiste básicamente en la utilización de datos masivos".

Gracias a las diversas aplicaciones y multifunciones que tiene el Big Data, este se traslada a diferentes medios, cual pueden ser los de comunicación social y también la publicidad (Campos, Rúas, López y Martínez, 2016).

\section{METODOLOGÍA}

El objeto de la presente investigación, de carácter aproximativo, consiste en efectuar un análisis de la evolución del marketing, desde el 1.0 al 4.0. La metodología aplicada se centra en la búsqueda de producción científica en la base de datos Scopus para el periodo comprendido entre el 2014 y 2018 . Se considera incluir 2018 como año a analizar por la reciente incorporación de la fase de marketing 4.0 .

Los dos objetivos planteados son:

- Analizar, de forma cuantitativa, la producción científica sobre Marketing, en sus diferentes fases, y Big Data.

- Conocer cuáles son las áreas de investigación con mayor producción científica en referencia al punto anterior en Scopus.

\section{RESULTADOS}

En este apartado se exponen los resultados a los objetivos planteados, el análisis de la producción científica de los términos Marketing, Marketing 1.0, Marketing 2.0, Marketing 3.0 y Marketing 4.0 y la combinación de Marketing, Marketing 3.0 y 4.0 con Big Data; y las áreas de mayor relevancia conforme a estos. Se determinan estos para el último quinquenio. 


\subsection{Comparativa de términos}

Realizada una revisión quinquenal de la literatura registrada en Scopus con relación a los términos seleccionados, se observa que del Marketing 2.0 ha sido el que más incidencia ha tenido en cuanto al interés mostrado para investigar sobre él, con un total de 342 artículos. De los demás términos, Marketing 1.0 (111), Marketing 3.0 (105) y Marketing 4.0 (128), se observa un porcentaje similar en cuanto a documentos registrados, entorno a cien, aunque parece que el Marketing 4.0 empieza a despuntar.

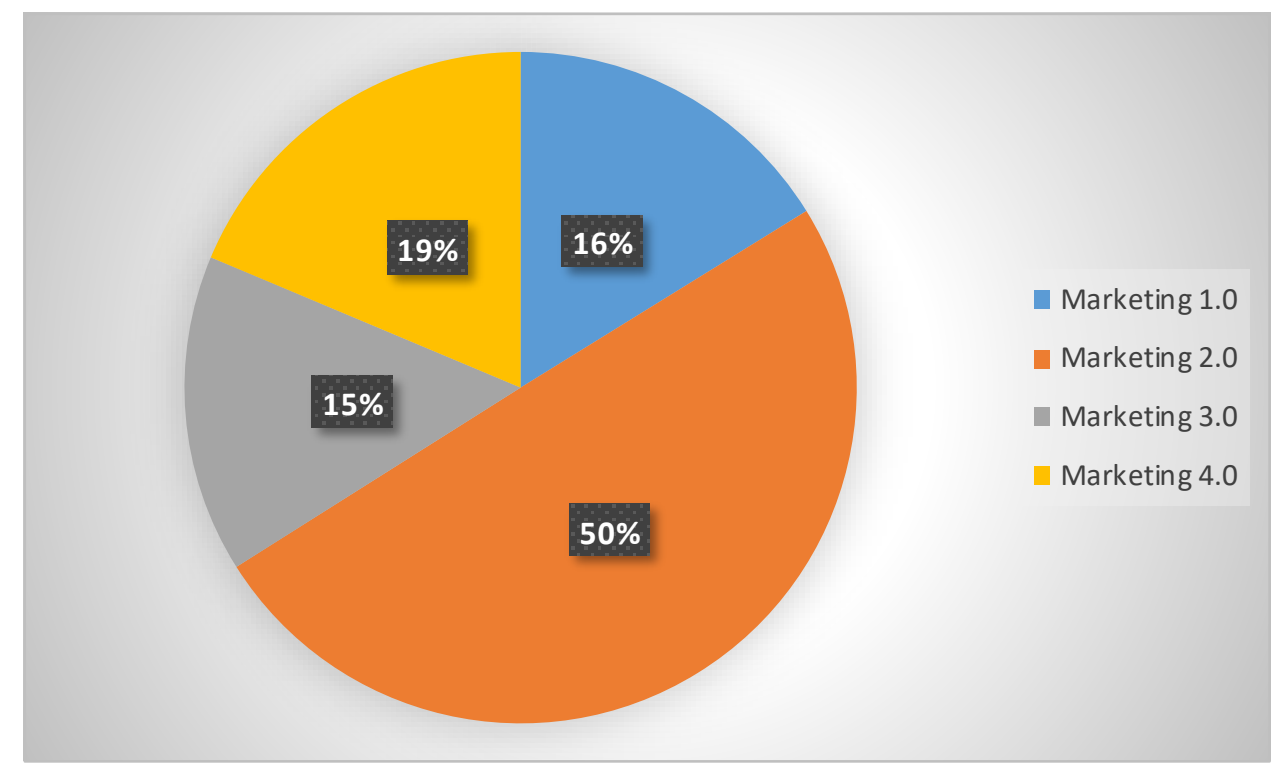

Fuente: elaboración propia a través de Scopus

Cabe destacar, que del término Marketing, en general, se han registrado un total de 53.480 documentos.

Por otra parte, si se combinan los términos de marketing, marketing 3.0 y 4.0 con Big Data, el número total de documentos registrados es de 865 , siendo 858 para Marketing \& Big Data y 3 y 4 para Marketing y Big Data 3.0 y 4.0 respectivamente.

\begin{tabular}{|l|ccccc|}
\cline { 2 - 6 } \multicolumn{1}{c|}{} & $\mathbf{2 0 1 4}$ & $\mathbf{2 0 1 5}$ & $\mathbf{2 0 1 6}$ & $\mathbf{2 0 1 7}$ & $\mathbf{2 0 1 8}$ \\
\hline Marketing \& Big Data & 94 & 155 & 196 & 203 & 210 \\
Marketing 3.0 \& Big Data & - & 1 & - & 1 & 1 \\
Marketing 4.0 \& Big Data & - & 1 & - & 2 & 1 \\
\hline
\end{tabular}

Fuente: elaboración propia a través de Scopus 


\section{2. Áreas de investigación más relevantes}

Si hablamos de Marketing en término general, se observa que el área de Business, Management and Accounting (Negocios, Gestión y Contabilidad) es el que se lleva la mayor producción científica con casi 19 mil documentos. El área de Medicine (Medicina) y Social Science (Ciencias Sociales) están más igualados en cuanto a publicaciones, entorno a los 10 mil. Quedando el Computer Science (Ciencias de la Computación) y Economics, Econometrics and Finance (Economía, Econometrías y Finanzas) más relegados, entre 6 y 8 mil.

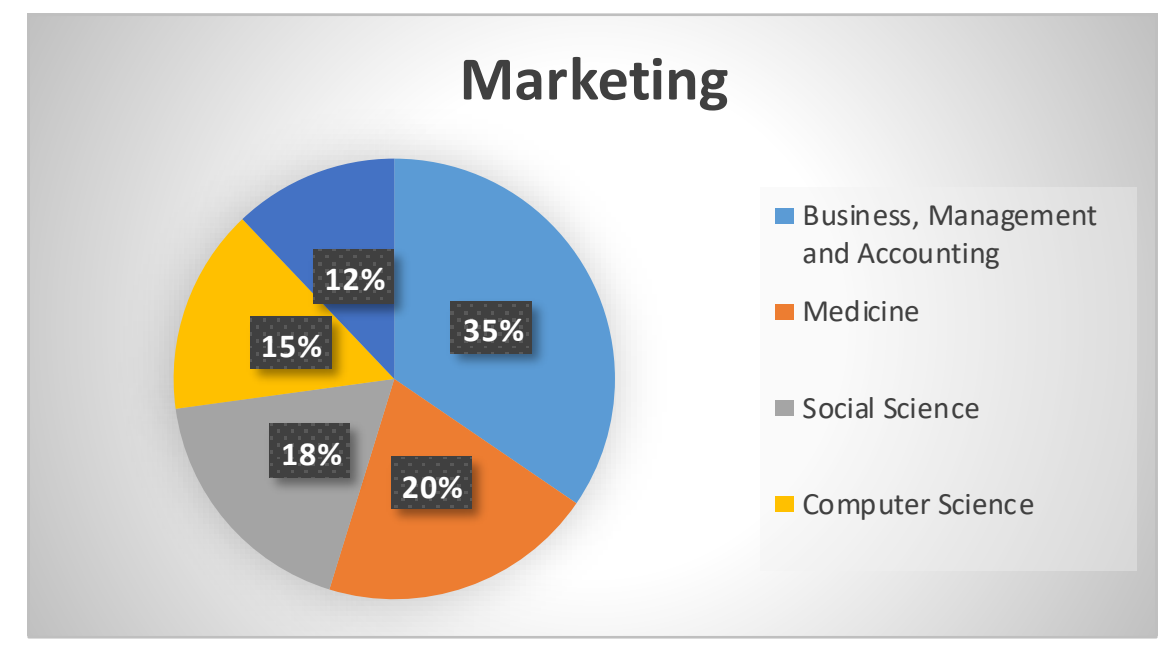

Fuente: elaboración propia a través de Scopus

Analizando las publicaciones en las distintas fases de la evolución del concepto de marketing, el Marketing 1.0 y 3.0 ha estado más centrado en el área de la medicina y otras de la misma rama como Agricultural and Biological Sciences (Ciencias Agrícolas y Biológicas) y Pharmacology, Toxicology and Pharmaceutics (Farmacología, Toxicología y Farmacia). 


\begin{tabular}{|lr|lr|}
\hline \multicolumn{1}{|c|}{ Marketing 1.0 } & \multicolumn{1}{c|}{ Marketing 3.0 } \\
\hline Medicine & 47 & Medicine & 38 \\
Agricultural and Biological Sciences & 26 & Business, Management and Accounting & 26 \\
Business, Management and Accounting & 12 & Economics, Econometrics and Finance & 18 \\
Pharmacology, Toxicology and Pharmaceutics & 11 & Agricultural and Biological Sciences & 17 \\
Social Science & 10 & Pharmacology, Toxicology and Pharmace & 14 \\
\hline
\end{tabular}

\begin{tabular}{|lr|lr|}
\hline \multicolumn{1}{|c|}{ Marketing 2.0 } & \multicolumn{1}{c|}{ Marketing 4.0 } \\
\hline Computer Science & 115 & Business, Management and Accounting & 40 \\
Business, Management and Accounting & 109 & Decision Sciences & 31 \\
Social Science & 78 & Medicine & 27 \\
Medicine & 56 & Agricultural and Biological Sciences & 20 \\
Economics, Econometrics and Finance & 44 & Computer Science & 15 \\
\hline
\end{tabular}

Fuente: elaboración propia a través de Scopus

La etapa de Marketing 2.0 está centrada en Computer Science y Business, Management and Accounting, sobrepasando los 100 documentos en ambos casos. Parece que esta tendencia se recuperará con el marketing 4.0 donde el Business, Management and Accounting destaca y Decision Sciences (Ciencias de la decisión) cobra protagonismo por primera vez en estos últimos 5 años.

Cuando se combinan las búsquedas con el término Big Data, ocurre lo mismo que en la etapa del Marketing 2.0, Computer Science y Business, Management and Accounting tienen más protagonismo, pero, Computer Science despunta con 523 documentos registrados en Scopus.

Según los datos extraídos, en la etapa del 3.0 y 4.0 junto al Big Data son los Negocios los que se llevan más atención, pero sin diferencia significativa.

\begin{tabular}{|lr|}
\hline \multicolumn{2}{|c|}{ Marketing \& Big Data } \\
\hline Computer Science & 523 \\
Business, Management and Accounting & 203 \\
Engineering & 178 \\
Mathematics & 148 \\
Social Sciences & 111 \\
\hline
\end{tabular}

Fuente: elaboración propia a través de Scopus

\begin{tabular}{|lr|}
\hline \multicolumn{1}{|c|}{ Marketing 3.0 \& Big Data } \\
\hline Business, Management and Accounting & 2 \\
Economics, Econometrics and Finance & 2 \\
Computer Science & 1 \\
Engineering & 1 \\
\hline
\end{tabular}

\begin{tabular}{|lr|}
\hline \multicolumn{2}{|c|}{ Marketing 4.0 \& Big Data } \\
\hline Business, Management and Accounting & 2 \\
Computer Science & 1 \\
Economics, Econometrics and Finance & 1 \\
Engineering & 1 \\
Materials Science & 1 \\
\hline
\end{tabular}




\section{DISCUSIÓN Y CONCLUSIONES}

La evolución que ha sufrido el marketing ha quedado manifiesta desde la época en la cual este apenas existía como tal, marketing 1.0, hasta la presente, marketing 4.0, en donde parece que se encuentra ligado al mundo digital. En este sentido, se ha visto como el marketing actual engloba un concepto mucho más amplio, donde importa tanto el mundo online como offline. Ahora tienen tanto que decir los clientes como las empresas y, lo más revelador, los clientes son escuchados realmente.

Estamos expuestos a una evolución vertiginosa de las tecnologías (5G, Inteligencia Artificial, Internet de las Cosas...) y del mercado, lo que hace inevitable estar pendientes de los posibles cambios que se produzcan y predecir a donde se dirigen.

Apoyarse en sistemas como el Big Data, el Internet de las Cosas y la Inteligencia Artificial puede ser un buen punto de partida para la "era de la predicción" que parece que pronto llegará. ¿Serán las maquinas quien conocerán antes que nosotros nuestros gustos? ¿Cuál será el potencial empresarial de la analítica predictiva para optimizar los gastos publicitarios y las decisiones de equipo? Si se consigue predecir el impacto financiero de los cambios en los precios o en los costes, ¿también se podrá cuantificar la repercusión de las recomendaciones de ofertas mejores a través de la satisfacción y fidelización de los clientes?

Si bien parece que el futuro aboga por un mundo donde los usuarios no han de pensar, no tienen que decidir las noticias para leer ni las series para ver, o que productos les interesan comprar (ya que de eso se encargará la predicción y el marketing), puede que se produzca algún nuevo punto de inflexión donde se cambie esta tendencia al igual que ocurrió, por ejemplo, en el paso del marketing 1.0 al 2.0, y se empezó a escuchar lo que los clientes tenían que decir. 


\section{REFERENCIAS BIBLIOGRÁFICAS}

American Marketing Association. (2018). Marketing Dictionary. Recuperado el $24 / 10 / 2018$

https://www.ama.org/resources/Pages/Dictionary.aspx?dLetter=M

Arroyo Figaredo, I. (2012). Un cliente insatisfecho comenta a una media de 10 personas su descontento. Consultado 24/10/2018 en Sitio web de Puro Marketing: http://www.puromarketing.com/14/11979/cliente-insatisfechocomenta-media-personas-descontento.html

Campos-Freire, F., Rúas-Araújo, J., López-García, X., \& Martínez-Fernández, V. A. (2016). Impacto de las redes sociales en el periodismo. El profesional de la información (EPI), 25(3), 449-457.

Carrizosa, S. (2014). Mike Walsh, Consejero Delegado De Tomorrow: "Los correos electrónicos y las páginas web tienen los días contados". El País. Consultado el 24/10/2018 en http://economia.elpais.com/economia/2014/12/05/actualidad/1417774968_0110 14.html

Chen, C. P. y Zhang, C. Y. (2014). Data-intensive applications, challenges, techniques and technologies: A survey on Big Data. Information Sciences, 275, 314-347.

De Vicuña Ancín, J. M. S. (2018). El plan de marketing digital en la práctica. ESIC Editorial.

Gutiérrez, J. A. T., Acebrón, L. B., y Casielles, R. V. (2005). Investigación de mercados: métodos de recogida y análisis de la información para la toma de decisiones en marketing. Editorial Paraninfo.

II Estudio Anual IAB Spain. TV Conectada y Video Online (2014). Consultado 4 de julio de 2018 http://www.iabspain.net/wpcontent/uploads/downloads/2014/11/II-Estudio-de-TV-Conectaa-yV\%C3\%ADdeo-Online-versi\%C3\%B3n-reducida.pdf 
Internet of the Things. (2015). En Gartner IT Glossary. Consultado 31/10/2018 en http://www.gartner.com/it-glossary/?s=internet+of+the+things

Kotler, P. (2001). Dirección de mercadotecnia: Análisis, planeación, implementación y control. Magíster en Administración-Tiempo Parcial 29, ESAN.

Kotler, P., Bowen, J., Makens, J. C., Moreno, R. R., y Paz, M. D. R. (2003). Marketing para turismo. McGraw-Hill.

Kotler, P., Kartajaya, H., y Setiawan I. (2013). Marketing 3.0: cómo atraer a los clientes con un marketing basado en valores. Madrid: LID Editorial Empresarial.

Kotler, P., Kartajaya, H., y Setiawan, I. (2010). Marketing 3.0: From products to customers to the human spirit: John Wiley \& Sons.

Kotler, P., Kartajaya, H., y Setiawan, I. (2017). Marketing 4.0. New Jersey, USA: John Wiley \& Sons.

Kotler, P., y Armstrong, G. (2003). Fundamentos de marketing. Pearson Educación.

Kotler, P., y Armstrong, G. (2008). Fundamentos de marketing Octava edición PEARSON EDUCACIÓN.

Kotler, P., y Armstrong, G. (2012). Principles of Marketing. New Jersey: Pearson Prentice Hall.

Krishnan, K. (2013). Data Warehousing in the Age of Big Data. Morgan Kaufmann.

Licklider, J. (1960). Man-Computer Symbiosis. Consultado 19/06/2018 http://groups.csail.mit.edu/medg/people/psz/Licklider.html

Marcotte, E. (2010). Responsive Web Design. Traducción al español por Marcelo Mazza. 25 de mayo de 2010. ( http://xn--diseowebresponsive-q0b.org/ ) 
Martin, J., y Ocampo, J. A. (2003). Globalización y desarrollo: una reflexión desde América Latina y el Caribe. Recuperado el 04/12/2018 de http://repositorio.cepal.org/bitstream/handle/11362/1811/S382104O15E_es.pdf ?sequence $=1$ \&isAllowed=y.

Martínez-Fernández, Valentín-Alejandro (2016). Immediacy and Metamedia. Time Dimension on Networks. PP. 19-24 En Media and Metamedia Management. Springer. Switzerland. ISBN: 978-3-319-46066-6 ISBN: 978-3-319-46068-0 eBook DOI: 10.1007/978-3-319-46068-0

Martin-Jiménez, M., (2016). Marketing digital. García Maroto, Madrid.

Mayer-Schönberger, V. y Cukier, K. (2013). Big Data. La revolución de los datos masivos. Madrid: Turner Publicaciones.

Mayer-Schönberger, V. y Cukier, K. (2013). Big Data. La revolución de los datos masivos. Madrid: Turner Publicaciones.

McAfee, A., Brynjonlfsson, E. (2012). Big Data: The Management Revolution. Hardvard Business Review, 90, 60-68

Montero, Y. H. (2015). Experiencia de usuario: principios y métodos. Experiencia de Usuario: Principios y Métodos, 9.

Morales, M. D. O., Aguilar, L. J., y Marín, L. M. G. (2016). Los desafíos del marketing en la era del big data. e-Ciencias de la Información, 6(1), 1-31.

Ordieres-Meré, J. (2014). Big Data E loT: claves del modelo de negocio para la empresa industrial del siglo XXI. Revista Economía Industrial, Ministerio de Industria, Energía y Turismo, Gobierno de España, 392, 113-122. Consultado el $31 / 10 / 2018$ http://www.minetur.gob.es/Publicaciones/Publicacionesperiodicas/Economialnd ustrial/RevistaEconomialndustrial/392/ORDIERES-MERÉ.pdf

PwC. (2015). Global Top 100 Companies by market capitalizatio. 31 March 2015 
Rouge, B. (2012). El papel de la serendipia en el descubrimiento de los efectos clínicos de los psicofármacos: más allá del mito. Actas Esp Psiquiatr, 40(1), 3442.

Salus, P. (1995). From ARPANET to Internet and Beyond. Boston, EEUU: Addison-Wesley Publishing.

Santesmases, M. (2012). Marketing. Madrid, España: Pirámide.

Sawhney, M. (2003). Fundamentals of Value. ClO Magazine, Jul, 1, 2003.

Schmarzo, B. (2014). Big Data: el poder de los datos. Anaya Multimedia.

Sixto, J. (2016). Fundamentos de Marketing Digital. Salamanca: Comunicación Social.

Tabares, L. F. y Hernández, J. F. (2014). Big Data Analytics: Oportunidades, Retos y Tendencias. Cali: Universidad de San Buenaventura.

\section{DE LA AUTORA}

\section{Tara Suárez-Cousillas}

Alumna de doctorado de la Universidade da Coruña (Facultad de Economía y Empresa) e Investigadora del Grupo Investigación Marketing Aplicado (iMarka) de la Universidade da Coruña (España). Más de 5 años de experiencia profesional en el ámbito de la Contabilidad y Fiscalidad. Graduada y Diplomada en Ciencias Empresariales. Máster en Contabilidad Superior y Auditoría de Cuentas Sus líneas de investigación se centran en el marketing digital y el consumo predictivo de contenidos. 